\title{
Absence of the first two zinc fingers in BCL6 causes the loss of inhibitory effects on cell growth
}

\author{
XIAOXING FU*, YING HAO*, YING MAO, YANLIN QIAN, ZUNLEI QIAN, \\ TING LI, DACHENG HE and XUEYUAN XIAO \\ Key Laboratory of Cell Proliferation and Regulation Biology, Ministry of Education, \\ Beijing Normal University, Beijing 100875, P.R. China
}

Received December 2, 2013; Accepted October 1, 2014

DOI: $10.3892 / \mathrm{mmr} .2015 .3185$

\begin{abstract}
BCL6ZF is a novel transcript of BCL6, which lacks the first two zinc fingers of BCL6. It has been established that BCL6 acts as a sequence-specific transcriptional repressor, however, the functions of $\mathrm{BCL} 6 \mathrm{ZF}$ remain undefined. By generating stably overexpressed BCL6 and BCL6ZF in NCI-H1299 lung cancer cells, it was found that BCL6 suppressed the levels of cell growth associated with impaired $G_{1}$ phase progression compared with those of the mock control cells. However, the effects of BCL6ZF on cell growth and the cell cycle were negligible. Further study of these results demonstrated that eight genes downstream of BCL6 were markedly downregulated by the overexpression of BCL6, whereas BCL6ZF suppressed only TGFBI, indicating that the loss of the first two zinc fingers caused the loss of the inhibitory effects on cell growth and transcriptional repression. In addition, it was determined that the BCL6ZF protein was not degraded as easily as BCL6 protein by the ubiquitin/proteasome pathway, implying that the loss of the first two zinc fingers changes the three-dimensional structure of BCL6ZF. The results demonstrated that BCL6 and BCL6ZF had different role in $\mathrm{H} 1299$ cells both in vitro and in vivo. The loss of its inhibitory effects on cell growth and transcriptional repressions.
\end{abstract}

\section{Introduction}

The BCL6 gene was originally identified in chromosomal translocations in non-Hodgkin lymphoma, and it acts as an

Correspondence to: Mr. Dacheng He or Miss. Xueyuan Xiao, Key Laboratory of Cell Proliferation and Regulation Biology, Ministry of Education, Beijing Normal University, 19 Xinjiekouwai Street, Beijing 100875, P.R. China

E-mail: dhe@bnu.edu.cn

E-mail: xyxiao@bnu.deu.cn

*Contributed equally

Key words: transcriptional repressor, BCL6, BCL6ZF, lung cancer important transcriptional repressor in lymphocyte proliferation and differentiation (1-5). BCL6 was also identified in a new subset of follicular $\mathrm{T}$ regulatory cells that localize to germinal centres (6-8). The BCL6 protein is composed of a POZ/BTB domain at the amino-terminal, six zinc finger $(\mathrm{ZF})$ motifs at the carboxyl-terminal and a PEST domain in the middle of the transcript (9). When BCL6 is overexpressed, it mediates apoptosis in U2OS, CV-1 and HeLa cells (10-12). Initial experiments prior to this study identified a novel transcript of BCL6, which had lost the first two zinc-fingers, that had been submitted to Genbank in 2008 with the name BCL6ZF (Accession no. EU139066). Notably, when comparing the sequence of BCL6ZF with that of the alternatively-spliced BCL6S, the latter lacked exon 7 and was identified from a human hippocampus cDNA library (DB465062) (13), although it was determined that they refer to the same transcript. While it was reported that BCL6S binds to the classical BCL6-binding sites and forms heterodimers with BCL6 (13-14), it is undetermined whether BCL6S has the same biological functions as BCL6.

In the current study, BCL6 and BCL6ZF were stably overexpressed in NCI-H1299 lung cancer cells and their biological functions were investigated.

\section{Materials and methods}

Cell culture and normal human tissue cDNA panels. The NCI-H1299, A549 and LTEP-a-2 human lung adenocarcinoma, NCI-H460 large cell lung cancer and NCI-H446 small cell lung cancer cell lines were maintained in the Key Laboratory of Cell Proliferation and Regulation Biology at Beijing Normal University (Beijing, China). The CL1-0, -1, -3 and -5 lung adenocarcinoma and NCI-H226 lung squamous carcinoma cell lines were gifted by Dr. Yi-Qing Wang and Dr. Gang Fang (National Taiwan Normal Universities, Taipei, Taiwan R.O.C.). All cell lines were cultured in RPMI-1640 medium (Gibco Life Technologies, Gaithersburg, MD, USA), supplemented with $10 \%$ fetal calf serum (FCS; HyClone, Logan, UT, USA) at $37^{\circ} \mathrm{C}$ in $5 \% \mathrm{CO}_{2}$. Normal tissue cDNA panels (multiple tissue cDNA panels I and II) comprising heart, brain, placenta, lung, liver, skeletal muscle, kidney, pancreas, spleen, thymus, prostate, testis, ovary, small intestine, colon and peripheral blood leukocytes were purchased from BD Biosciences Clontech (Palo Alto, CA, USA). 
RNA isolation and reverse transcription. Total RNA was isolated from cell lines using TRIzol Reagent (Invitrogen Life Technologies, Carlsbad, CA, USA), and $2 \mu \mathrm{g}$ of RNA was reverse transcribed into cDNA with Molony murine leukaemia virus reverse transcriptase (Promega, Madison, WI, USA) and oligo (dT)15 (Promega) as a primer.

PCR reactions were carried out with BCL6 specific primers flanking the first two zinc fingers (forward, 5'-GAG TGATTCGAGTGTGGGTT-3', reverse, 5'-ATGGAGCCT GAGAACCTTGA-3') or with $\beta$-actin specific primers (forward, 5'-GAGCTACGAGCTGCCTGACG-3', reverse, 5'-CCTAGAAGCATTTGCGGTGG-3') or GAPDH specific primers (forward, 5'-TCAACGGATTTGGTCGTATTG-3', reverse, 5'-TGGAAGATGGTGATGGGATT-3') as an internal control. All PCR reactions consisted of an initial denaturation at $94^{\circ} \mathrm{C}$ for $5 \mathrm{~min}$ followed by 25 (for actin) or 28 cycles (for BCL6) of: $94^{\circ} \mathrm{C}$ for $30 \mathrm{sec}, 56^{\circ} \mathrm{C}$ for $30 \mathrm{sec}$ and $72^{\circ} \mathrm{C}$ for $60 \mathrm{sec}$ with a final extension step at $72^{\circ} \mathrm{C}$ for 10 min, all using an ABI GeneAmp PCR System 9700 (Applied Biosystems Life Technologies, Foster City, CA, USA). Equal volumes of each PCR sample were analysed by electrophoresis on a $1 \%$ agarose gel following ethidium bromide staining.

Generation of cell lines stably expressing BCL6 and BCL6ZF. Cells were cultured to $80 \%$ confluence, and then NCI-H1299 cells were transfected with either the BCL6/pcDNA3.1, BCL6ZF/pcDNA3.1 or pcDNA3.1 empty plasmid, by using FuGENE 6 transfection reagent (Roche, Indianapolis, IN, USA) according to the manufacturer's instructions. Following a 6-h transfection, $600 \mu \mathrm{g} / \mathrm{ml} \mathrm{G418-sulfate} \mathrm{(G418;} \mathrm{Merck}$ Millipore, Darmstadt, Germany) was added to the medium. Following 2 weeks of G418 treatment, resistant clones were confirmed by RT-qPCR and western blot analysis and selected for further study.

In vitro cell proliferation assay. Cell proliferation was assessed using a Z2 Coulter Counter ${ }^{\circledR}$ Cell and Particle Counter (Beckman Coulter, Brea, CA, USA). Each cell line (1 ml, $3 \times 10^{4}$ cells) was seeded into 24 -well plates. Cell samples were trypsinized and counted with the cell counter, three samples a day, for $\sim 1$ week. The numbers were recorded and used to construct growth curves.

Cell cycle analysis. The cells were trypsinized and fixed in $70 \%$ ice-cold ethanol for at least $24 \mathrm{~h}$, prior to centrifugation for $5 \mathrm{~min}$ at $125 \mathrm{x} \mathrm{g}$, and resuspension in $200 \mu \mathrm{l}$ PBS. RNase A and propidium iodide were added to a final concentration of $50 \mu \mathrm{g} / \mathrm{ml}$ and $10 \mathrm{mM}$, respectively, and the cells were incubated on ice for $4 \mathrm{~h}$ prior to analysis with a FACSCalibur flow cytometer (BD Biosciences, Franklin Lakes, NJ, USA). A total of 10,000 events were analysed for each sample.

Tumorigenesis in vivo. For tests in vivo, animal studies were performed. A total of $18 \mathrm{BALB} / \mathrm{C}$ nude mice were acquired by the Perking University (Beijing, China). The animal studies were approved by the Ethics committee of College of Life Science (Beijing Normal University). All methods were carried out according to institutional guidelines. BCL6/BCL6ZF transfected cells and control cells were collected at a concentration of $5 \times 10^{6}$ cells per $100 \mu \mathrm{l}$ and injected into 4-week-old male nude mice, with six mice per transfected or control cell line. Tumor volumes were measured every 3 days, and calculated using the equation: $V\left(\mathrm{~mm}^{3}\right)=\left(a \times b^{2}\right) / 2$, where $a$ is the largest diameter and $b$ is the perpendicular diameter. The tumors were weighed following necropsy.

Quantitative PCR ( $q P C R)$. Specific primers (Table I) were used for qPCR. The reactions were performed with the ABI 7300 Real-Time PCR System and SYBR ${ }^{\circledR}$ Green (Applied Biosystems Life Technologies), according to the manufacturer's instructions. GAPDH was used as an internal control. Each experiment was performed in a total volume of $20 \mu \mathrm{l}$, containing $10 \mu \mathrm{l} \mathrm{SYBR}{ }^{\circledR}$ Green PCR Master Mix (Applied Biosystems), $1 \mu \mathrm{l}$ cDNA or $\mathrm{H}_{2} \mathrm{O}$ as negative control, $1 \mu \mathrm{l}$ each of forward and reverse primers $(10 \mathrm{mM})$ and $7 \mu \mathrm{l}$ distilled $\mathrm{H}_{2} \mathrm{O}$. The thermal cycling conditions were as follows: $95^{\circ} \mathrm{C}$ for $10 \mathrm{~min}$, followed by 40 cycles of $95^{\circ} \mathrm{C}$ for $30 \mathrm{sec}$ and $60^{\circ} \mathrm{C}$ for $1 \mathrm{~min}$. Uniform amplification of the product was confirmed by analysing the melting curves of the amplified products. All reactions were carried out in triplicate to assess the reproducibility. The relative quantification of mRNA expression was calculated by the comparative $\mathrm{Ct}$ (threshold cycle) method using the follow formula:

$$
\text { Ratio }=2^{-\Delta \Delta C t}=2^{-[\Delta C t(\text { sample })-\Delta C t(\text { calibrator })]}
$$

where $\Delta C t=C t$ of target genes - $C t$ of endogenous control gene $(\mathrm{GAPDH})$.

Western blot analysis. Equal quantities of protein were separated by $10 \%$ SDS-PAGE and transferred onto a nitrocellulose membrane (GE Healthcare Life Sciences, Chalfont, UK) using a Bio-Rad wet transfer unit. Following blocking with $5 \%(\mathrm{w} / \mathrm{v})$ non-fat dried milk in a tris-buffered saline with Tween 20 (TBST) solution [25 mMTris, pH7.5, $150 \mathrm{mM} \mathrm{NaCl}, 0.05 \%$ (v/v) Tween 20] for $1 \mathrm{~h}$ at room temperature, the membranes were incubated with mouse anti-human BCL6 monoclonal antibody (dilution with TBST, $1: 100)$ and mouse anti-human $\beta$-actin antibody $(1: 1,000)$ for $1 \mathrm{~h}$ at $37^{\circ} \mathrm{C}$ or overnight at $4^{\circ} \mathrm{C}$, followed by alkaline phosphatase-conjugated horse anti-mouse $\operatorname{IgG}(1: 1,000)$ for $1 \mathrm{~h}$ at $37^{\circ} \mathrm{C}$. All antibodies were obtained from Santa Cruz Biotechnology, Inc. (Dallas, TX, USA). Target proteins were detected with 5-bromo-4-chloro-3-indoyl phosphate (BCIP) and nitro blue tetrazolium (NBT). $\beta$-actin was chosen as an internal control for equal protein loading. In case where the cells needed to be treated with the proteasome inhibitor MG132, the cells were incubated with $50 \mu \mathrm{M}$ MG132 for $12 \mathrm{~h}$ and the proteins were extracted from the cells.

Statistical analysis. SPSS statistical software version 16.0 (SPSS, Inc., Chicago, IL, USA) was used to conduct all statistical analysis. Fisher's exact test and Student's t test were used for the comparison between the tumors with BCL6 or BCL6ZF expression and the control. For a simple comparison of two values, the Student's t test was used where appropriate. All statistical tests were two-tailed. $\mathrm{P}<0.05$ was considered to indicate a statistically significant difference. 
Table I. Primers used for quantitative polymerase chain reaction.

\begin{tabular}{ll}
\hline Gene & \multicolumn{1}{c}{ Sequence (5'-3') } \\
\hline KRT8 & S: CAGTACGAGGATATTGCCACCCG \\
& A: CAGTCTTTGTGAGCGCAGGTC \\
RND3 & S: TCCATGTGTTCGCCAAGGACTGC \\
& A: CCGAAGTGTCCACAGGCTCAAC \\
TGFbI & S: CCGTTGATAGTGAGCATGTCCC \\
& A: CTTCGAGAAGATCCCTAGTGAGA \\
ANXA1 & S: CTAAGGGTGACCGATGTGAGGAC \\
& A:TGGATAGGTTCTGGTGGTAAGGA \\
COX7B2 & S: CCTATCCCCTGTTGGCAGAGTT \\
& A: AGTGCTTACACGACAAGTTGGTT \\
THBS1 & S: CTGACTGGCGTTAGCCGATTA \\
& A: GCAGGTGGTGAGTAAGGGTGGG \\
PDCD5 & S: CATGGCGGACGAGGAGGTTGAG \\
& A: TTTCTGCTTCCCTGTGCTTTGC \\
RELB & S: GAAAGACTGGACCGACGACATCT \\
& A: TTCCGCTCAATGTCAGCTTCAAT \\
MIP1a & S: AGCTGGTTTCAGACTTCAGTAGGAC \\
& A: TGATTCTGAGCAGGTGACGGAA \\
PRDM1 & S: AACCTGGCTGCGTGTCAGAAC \\
& A: CTCGGTTGCTTTAGACTGCTCTG \\
GAPDH & S: GAGTCAACGGATTTGGTCGT \\
& A: GACAAGCTTCCCGTTCTCAG \\
\end{tabular}

$\mathrm{S}$, sense; A, antisense.

\section{Results}

Discovery and distribution of BCL6ZF. In a previous study, which tested BCL6 expression levels in lung cancer tissues and paired adjacent noncancerous tissues, it was determined that not only did BCL6 have discrepant expression between these two groups, but notably, two different sizes of PCR products were detected by RT-PCR using the BCL6 primers (data not shown). Furthermore these two PCR products were cloned and sequenced. The results revealed that the larger fragment was the normal full-length transcript of BCL6, while the shorter one lacked exon 7 (168 bp) revealing that it had lost the first two zinc-fingers of BCL6 and it was named BCL6ZF (GenBank Accession No. EU139066). However, it was not clear whether BCL6ZF, like BCL6, could be expressed.

In the current study, 16 normal human tissue samples and ten lung cancer cell lines were examined to investigate the distributions of BCL6ZF. As expected, BCL6ZF was found in all the tested samples but brain and skeletal muscle, which only expressed BCL6 (Fig. 1).

BCL6 and BCL6ZF have different effects on cell growth. To explore the effects of BCL6 and BCL6ZF on lung cancer, the NCI-H1299 cell line was selected for transfection with BCL6 or BCL6ZF vectors due to its lower endogenous expression levels compared with those of the other cell lines that were tested. Compared with the mock control cells, NCI-H1299 cells expressing BCL6 showed significantly suppressed cell growth and delayed $\mathrm{G}_{1}$ phase progression. However, no sub- $\mathrm{G}_{1}$ phase was observed, implying that the cell growth suppression caused by the overexpression of BCL6 was not associated with apoptosis. Unexpectedly, overexpression of BCL6ZF did not have similar effects to BCL6, as neither cell growth nor the cell cycle were altered markedly (Fig. 2A-B), indicating that the negative control of BCL6 on cell growth is associated with the first two zinc-fingers.

In agreement with the results in vitro, the tumors derived from NCI-H1299 cells expressing BCL6 grew significantly slower than those with endogenous BCL6 expression $(\mathrm{P}<0.05)$. However, no obvious differences in tumor growth were observed between the BCL6ZF overexpression and the control groups (Fig. 2C). The average tumor weight in the BCL6 group was $0.48 \pm 0.21 \mathrm{~g}$, which was significantly lighter than that of the BCL6ZF expression $(1.80 \pm 0.45 \mathrm{~g})$ and control groups $(1.33 \pm 0.46 \mathrm{~g}, \mathrm{P}<0.05)$.

Difference in the transcriptional repression ability of BCL6 and $B C L 6 Z F$. To confirm whether the loss of the growth inhibition ability of BCL6ZF is associated with the loss of transcriptional repression, eight BCL6-regulated genes were selected depending on the results of DNA arrays (data not shown) and further tested using qPCR. All of the tested genes were found to have markedly reduced expression levels in the BCL6 group, compared with those in the control group, whereas only TGFBI was repressed in BCL6ZF group (Fig. 3A), suggesting that BCL6ZF may have an innovative functions in regulating transcription. To validate this hypothesis, two typical BCL6 target genes, PRDM1 and MIPla, were selected and analysed by qPCR. It was determined that the expression of PRDM1 was not markedly repressed in the BCL6ZF group (Fig. 3B). Based on these results, it is reasonable to hypothesize that the loss of the first two zinc-fingers of BCL6 not only impairs DNA binding, but may additionally affect interactions with other co-repressors, leading to indirect suppression of the transcription of the BCL6 target gene.

$B C L 6 Z F$ was not readily degraded via the ubiquitin/proteasome pathway, as compared with BCL6. In the present study, it was noted that the ectopic BCL6ZF proteins were expressed and easily detected, whereas both of the endogenous and the ectopic BCL6 proteins were barely detected (Fig. 4A), implying that the BCL6ZF protein may have structural changes or some other unknown influences resulting the protein being less degraded.

To validate these hypotheses, the cells were incubated with the proteasome inhibitor MG132. As expected, the expression level of the BCL6 protein increased much more than those of the BCL6ZF protein in cells treated with MG132 (Fig. 4B). These results indicate that loss of the first two zinc fingers may give rise to three-dimensional structural changes in BCL6ZF so that it is not degraded as easily as BCL6 by the proteasome.

\section{Discussion}

The zinc-finger region of BCL6 is considered to have multiple functions; besides its DNA binding ability, it is involved in 


\section{A}

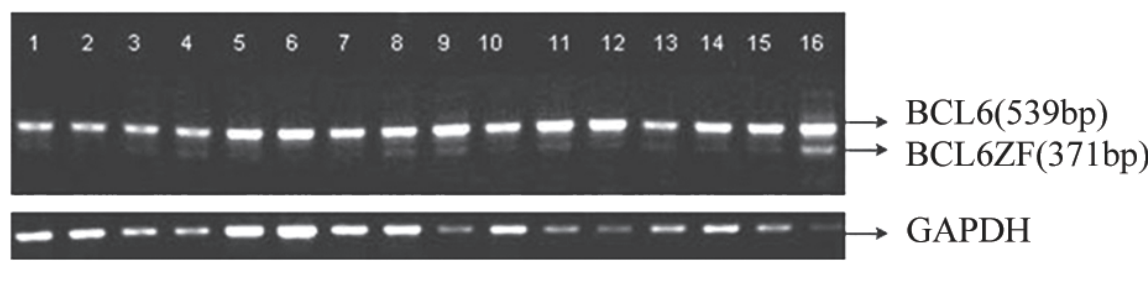

B

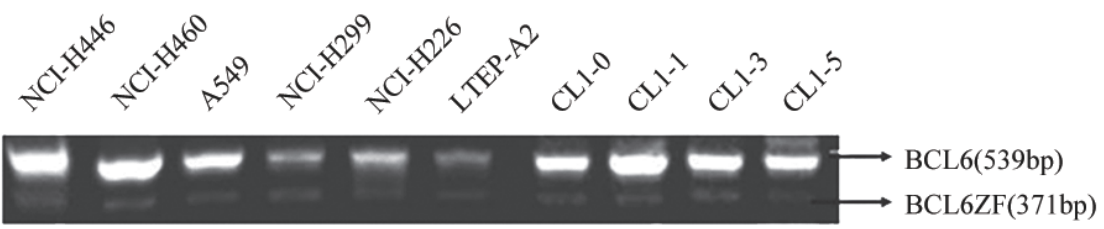

C
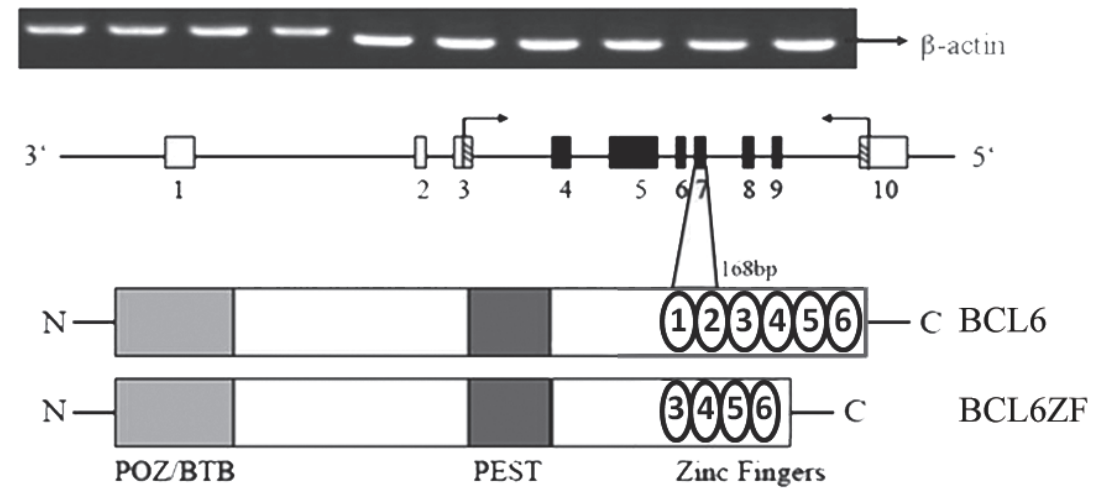

Figure 1. Expressions levels of BCL6 and BCL6ZF were analyzed by reverse transcription polymerase chain reaction using primers flanking the two zinc finger regions, resulting in $539 \mathrm{bp}$ and $371 \mathrm{bp}$ fragments corresponding to BCL6 and BCL6ZF. GAPDH was used as a control. Expression levels of BCL6 and BCL6ZF in (A) sixteen normal human tissues. Lane 1: heart, 2: brain, 3: placenta, 4: lung, 5: liver, 6: skeleton muscle, 7: kidney, 8: pancreas, 9: spleen, 10: thymus, 11: prostate, 12: testis, 13: ovary, 14: small intestine, 15: colon, 16: peripheral blood and (B) ten lung cancer cell lines. (C) The gene and protein structures of BCL6 and BCL6ZF.

A

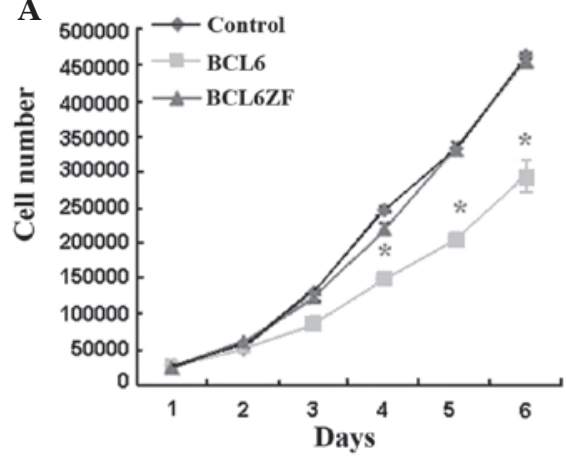

C

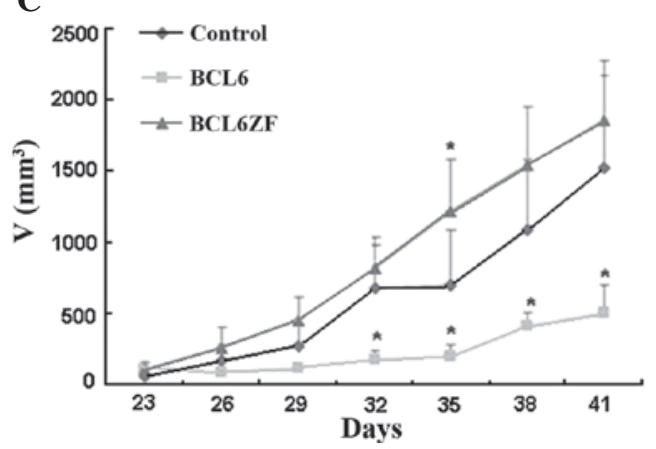

B
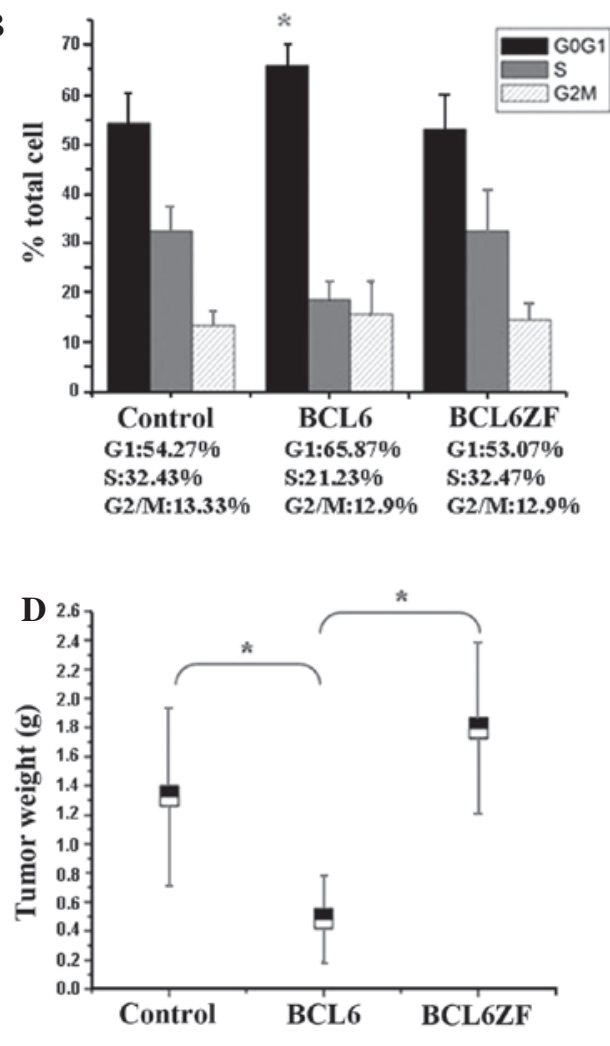

Figure 2. Effects of the overexpression of BCL6ZF and BCL6 on cell proliferation. (A) The growth curves of cells transfected with BCL6 and BCL6ZF were compared with the vector cells using a Z2 Coulter Counter ${ }^{\circledR}$ Cell and Particle Counter. (B) The cell cycle was analyzed using flow cytometry. (C) The volumes of xenograft tumors derived from BCL6 and BCL6ZF overexpression were measured every three days and compared with control groups. (D). Xenograft tumors were weighed following necropsy. Values represent the means \pm standard deviation of at least three independent experiments, ${ }^{\prime} \mathrm{P}<0.05$. 
A

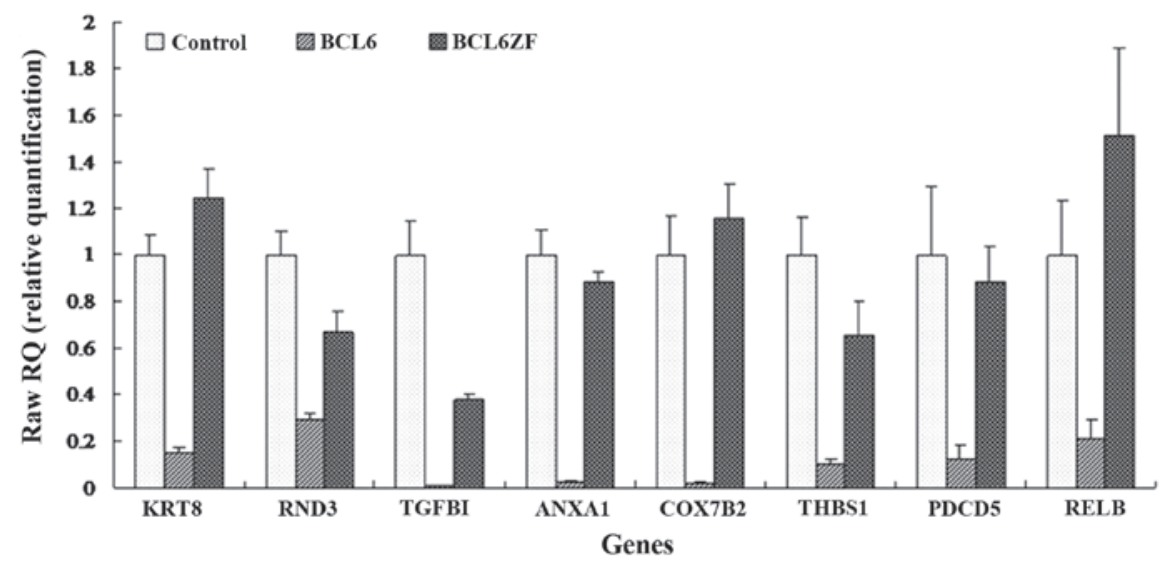

B

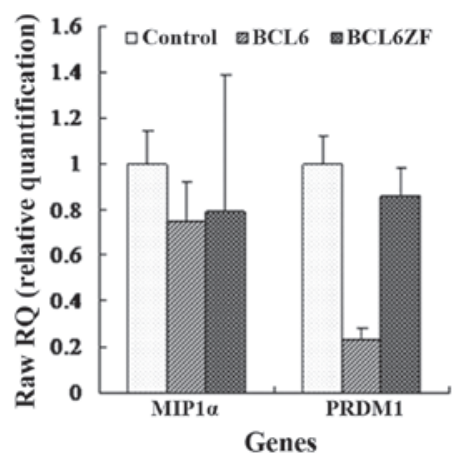

Figure 3. The transcriptional repression ability of BCL6 and BCL6ZF. (A) Quantitative polymerase chain reaction (qPCR) validation of eight genes regulated by BCL6. (B) Two BCL6 reported target genes expression analysis by qPCR. Samples were analyzed in triplicate and results represent the mean \pm standard error of the mean.

A

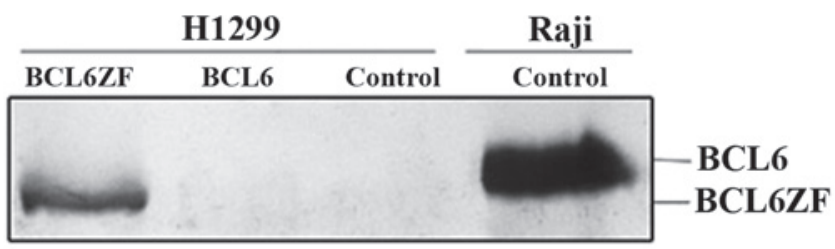

B
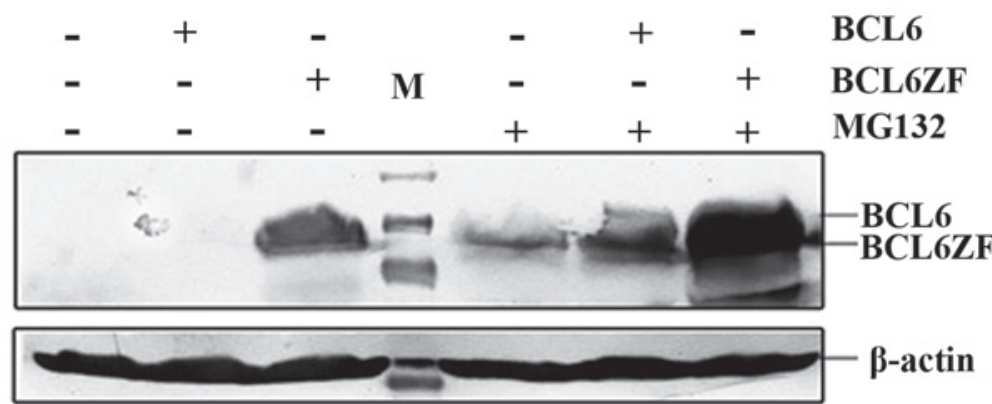

Figure 4. Degradation of BCL6 and BCL6ZF through the ubiquitin/proteasome pathway. (A) The expression levels of BCL6 and BCL6ZF were analyzed in stably transfected NCI-H1299 cells by western blot analysis, with Raji cells as a control. (B) Western blot analysis of BCL-6 and BCL6ZF proteins in stably transfected NCI-H1299 cells with or without $50 \mu \mathrm{M}$ MG132 (added $12 \mathrm{~h}$ ). M, marker.

the recruitment of the class II HDACs and interactions with a number of other proteins (15-16). However, point mutation of the two N-terminal zinc-fingers of BCL6 did not impair DNA binding, protein localization or the transcriptional repression function, indicating that these first two zinc-finger regions may not be as important compared with the others (17). It was previousl reported that similar to BCL6, overexpression of
BCL6S repressed four BCL6 typical target genes, including PRDM1 and MIP1a, in HEK 293T cells, based on the results of luciferase reporter assays (13). These results indicate that the first two zinc-fingers were not necessary for the defining functions of BCL6.

In the present study, it was determined that BCL6ZF, unlike BCL6, not only lost the growth-suppressing effects 
on lung cancer cells in vitro and in vivo, but in addition, it differed from BCL6 in how easily it was degraded via the ubiquitin/proteasome pathway. To further investigate the differences between BCL6 and BCL6ZF, Illumina Gene Chips (human WG-6-v3-0-R2-11282955-A; San Diego, CA, USA) were used to discover the target or downstream genes regulated by BCL6 and BCL6ZF. A total of 256 genes were identified to have differential expression (+/-2 fold change) between the BCL6 overexpression and control groups, whereas only eight genes showed a marked change in expression levels between the BCL6ZF and control groups (data not shown). To confirm the changes in the expression profiles detected by the microarray, eight differential genes were selected and their expression levels were analysed by RT-qPCR. As expected, the results were consistent with the microarrays, indicating that BCL6ZF lost the DNA binding capacity. This hypothesis was further confirmed by analysing the PRDM1 expression level, which is one of the BCL6 target genes. This discordance with BCL6S may be due to the different cell type, transfection and analysing methods used. In conclusion, our preliminary results suggested that the first two zinc-fingers of BCL6 may have a very important role in its function, and that BCL6ZF may have structural changes or lose its interaction with other corepressors. To clarify this matter, the crystal structure or promoter array analysis of BCL6ZF is required.

Our results suggest that BCL6ZF may antagonize or compete the role of BCL6 in lung cancer cells and a decreased expression of BCL6ZF may be helpful to treat lung cancer patients in clinic.

\section{Acknowledgements}

This study was supported by the Ministry of Science and Technology of China (no. 2010CB912203 and 2011CB915504), of the National Natural Science Foundation of China (no. 81272604 and 81172216).

\section{References}

1. Ye BH, Lista F, Lo Coco F, et al: Alterations of a zinc finger-encoding gene, BCL-6, in diffuse large-cell lymphoma. Science 262: 747-750, 1993.
2. Jardin F, Ruminy P, Bastard C, et al: The BCL6 proto-oncogene: a leading role during germinal center development and lymphomagenesis. Pathol Biol 55: 73-83, 2007.

3. Chang CC, Ye BH, Chaganti RS and Dalla-Favera R: BCL-6, a $\mathrm{POZ/zinc-finger} \mathrm{protein,} \mathrm{is} \mathrm{a} \mathrm{sequence-specific} \mathrm{transcriptional}$ repressor. Proc Natl Acad Sci USA 93: 6947-6952, 1996.

4. Morin RD, Mendez-Lago M, Mungall AJ, et al: Frequent mutation of histone-modifying genes in non-Hodgkin lymphoma. Nature 476: 298-303, 2011.

5. Pasqualucci L, Dominguez-Sola D, Chiarenza A, et al: Inactivating mutations of acetyltransferase genes in B-cell lymphoma. Nature 471: 189-195, 2011.

6. Nurieva RI, Chung Y, Martinez GJ, et al: Bcl6 mediates the development of T follicular helper cells. Science 325: 1001-1005, 2009.

7. Johnston RJ, Poholek AC, DiToro D, et al: Bcl6 and Blimp-1 are reciprocal and antagonistic regulators of $\mathrm{T}$ follicular helper cell differentiation. Science 325: 1006-1010, 2009.

8. Chung Y, Tanaka S, Chu F, et al: Follicular regulatory T cells expressing Foxp3 and Bcl-6 suppress germinal center reactions. Nat Med 17: 983-988, 2011.

9. Margalit O, Amram H, Amariglio N, et al: BCL6 is regulated by p53 through a response element frequently disrupted in B-cell non-Hodgkin lymphoma. Blood 107: 1599-1607, 2006.

10. Albagli O, Lantoine D, Quief S, et al: Overexpressed BCL6 (LAZ3) oncoprotein triggers apoptosis, delays $\mathrm{S}$ phase progression and associates with replication foci. Oncogene 18: 5063-5075, 1999.

11. Yamochi T, Kaneita Y, Akiyama T, et al: Adenovirus-mediated high expression of BCL-6 in CV-1 cells induces apoptotic cell death accompanied by down-regulation of BCL-2 and BCL-XL. Oncogene 18: 487-494, 1999.

12. Salamon D, Adori M, He M, et al: Type I interferons directly down-regulate BCL-6 in primary and transformed germinal center B cells: differential regulation in B cell lines derived from endemic or sporadic Burkitt's lymphoma. Cytokine 57: 360-371, 2012.

13. Shen Y, Ge B, Ramachandrareddy H, et al: Alternative splicing generates a short BCL6 (BCL6S) isoform encoding a compact repressor. Biochem Biophys Res Commun 375: 190-193, 2008.

14. Duan S, Cermak L, Pagan JK, et al: FBXO11 targets BCL6 for degradation and is inactivated in diffuse large B-cell lymphomas. Nature 481: 90-93, 2012.

15. Lemercier C, Brocard MP, Puvion-Dutilleul F, et al: Class II histone deacetylases are directly recruited by the BCL6 transcriptional repressor. J Biol Chem 277: 22045-22052, 2002.

16. Miles RR1, Crockett DK, Lim MS and Elenitoba-Johnson KS.: Analysis of BCL6-interacting Proteins by Tandem Mass Spectrometry. Mol Cell Proteomics 4: 1898-1909, 2005.

17. Mascle $\mathrm{X}$, Albagli $\mathrm{O}$ and Lemercier C: Point mutations in BCL6 DNA-binding domain reveal distinct roles for the six zinc fingers. Biochem Biophys Res Commun 300: 391-396, 2003. 